Research Article

\title{
Optimisation Analysis of Structural Parameters of an Annular Slot Ejector Based on the Coanda Effect
}

\author{
Fengliang $\mathrm{Wu} \mathbb{D}^{1}$ and Zhisheng $\mathrm{Li} \mathbb{D}^{1,2}$ \\ ${ }^{1}$ College of Safety Science and Engineering, Xi'an University of Science and Technology, Xi'an 710054, China \\ ${ }^{2}$ Faculty of Geosciences and Environmental Engineering, Southwest Jiaotong University, Chengdu 610031, China \\ Correspondence should be addressed to Fengliang Wu; wufl@xust.edu.cn
}

Received 13 March 2020; Revised 2 July 2020; Accepted 16 July 2020; Published 11 August 2020

Guest Editor: Sanghyuk Lee

Copyright (C) 2020 Fengliang Wu and Zhisheng Li. This is an open access article distributed under the Creative Commons Attribution License, which permits unrestricted use, distribution, and reproduction in any medium, provided the original work is properly cited.

\begin{abstract}
To investigate the effect of structural parameters on the performance of an annular slot ejector, a series of numerical simulations were conducted with single-factor analysis. Moreover, a multifactor grey relational analysis was applied to examine the correlations between the structural parameters and entrainment ratio. Subsequently, the optimised model was verified by comparing the simulated results with experimental data. Results show that the performance of the optimised ejector model was improved. The RNG $k-\varepsilon$ turbulent transport mode can simulate the internal field characteristics of an annular slot ejector, and the corresponding simulated results, as verified by experiment, satisfy engineering requirements. In addition, a quantitative correlation between structural parameters and entrainment ratio was obtained as follows: $e$ (nozzle clearance) $>\theta$ (diffusing chamber angle) $>H_{L}$ (mixing chamber length) $>d$ (throat diameter) $>K_{L}($ diffusing chamber length). This work may provide a certain guiding significance for the design and application of annular slot ejectors.
\end{abstract}

\section{Introduction}

An annular slot ejector is a mechanical device which is different from a central jet [1-4]. A large volume of lowpressure fluid is dragged by high-pressure fluid due to the special structure of the annular slot ejector [5]. The working principle of an annular slot ejector is known as the Coanda effect $[6,7]$. The phenomenon is described as a fluid flowing not along the original direction but along a curved surface. Subsequently, the mainstream flow passes through the nozzle clearance, it deflects and flows along the wall surface, and then induces a large amount of air from its surroundings [8]. Gregory-Smith and Gilchrist described three main features of the Coanda effect including the nonviscous effect, viscous effect, and a stronger entrainment capability [9]. Besides, another major feature of the Coanda effect is the Coanda flare, also known as a shock wave when the high-pressure gas reaches a certain speed (Figure 1).
The Coanda effect has received extensive attention in aviation [11], medicine [12], acoustics [8], robotics [13], and leafless fans [14]. Dong-Won et al. utilised a Coanda nozzle to cause jet deflection and wall shearing [15]. Their work showed that when the pressure increases, the potential splash zone tends to move downstream. In recent years, the introduction of the Coanda effect has promoted research into, and development of, annular ejectors [10, 16-25].

Ameri proposed a semiempirical formula for the section velocity based on a new ejector model by conducting a set of experiments with an LDV (laser Doppler velocimeter) [26]. In his study, it was assumed that the flow between the pressure inlet and the nozzle must satisfy the isentropic condition; however, the isentropic condition cannot be applied at high pressures. Guerriero investigated the influence of structural parameters on the ejector performance by conducting a set of experiments. They suggested that nozzle clearance has a significant influence on the ejector performance [27] and the corresponding results under the 


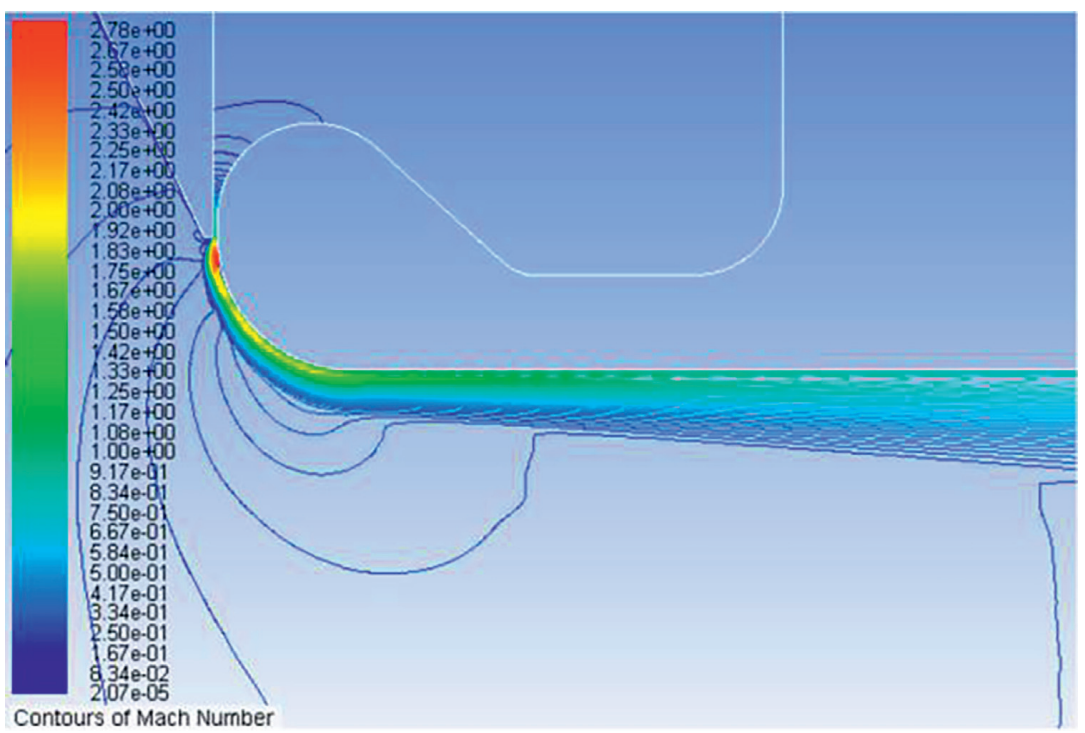

(a)

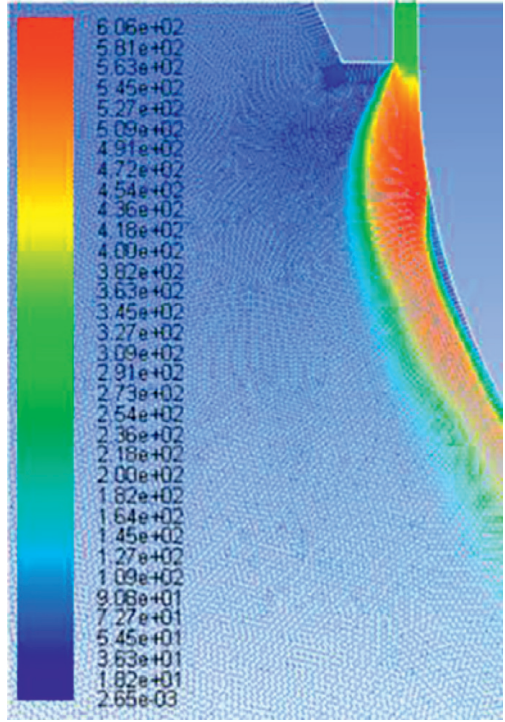

(b)

Figure 1: A Coanda flare (a) contours of Mach number at the ejector throat and (b) contours of velocity [10].

same model and working conditions were verified by Kim et al. employing CFD methods. Moreover, they pointed out that the stagnation pressure ratio is another important parameter affecting ejector performance $[28,29]$. Alexandru et al. developed a semiempirical formula for two-dimensional Coanda flow while the curvature of the tangential momentum equation was neglected [16]. Sierra del Rio et al. designed a two-ejector model with varying nozzle clearance $(0.3 \mathrm{~mm}$ and $0.8 \mathrm{~mm})$ to investigate the effect of nozzle clearance on the flow velocity by means of CFD methods [30]; their results showed that the velocity increases with the increase of nozzle clearance. Similar trends were obtained by Lowry et al. based on a new Coanda ejector [31]. Jain et al. developed a new ejector model with two nozzles to investigate the relationship between the structural parameters (nozzle clearance and throat diameter) and the flow characteristic. They also suggested that the nozzle clearance has a significant influence on the flow velocity, and the larger the throat diameter is, the faster the mixing layer is developed [10].

Although many studies have been conducted, previous studies focused on single-factor analysis and few studies, where all structural parameters were varied simultaneously, have been undertaken. Moreover, different optimal sizes of the model were obtained due to the differences in the structure being modelled. Besides, in the early literature only the nozzle clearance is deemed to have been an important parameter that influences ejector performance, but correlations between other geometric parameters and the entrainment ratio were ignored. In the present work, a set of numerical simulations were conducted using single-factor analysis and multifactor analysis to investigate annular slot ejector performance including five structural parameters (namely, mixing chamber length, diffusion chamber length, diffusion chamber angle, throat diameter, and nozzle clearance). Moreover, the optimised model was verified and analysed by conducting a series of experiments to compare with the results of numerical simulation.

\section{Numerical Model}

2.1. Turbulent Model. The flow of gas inside the annular ejector contains turbulence, and the velocity gradient of the mainstream gas at the throat of the ejector changes significantly which may generate more vortices. Amel et al. analysed the variation of the flow characteristic for both single-phase flow and two-phase flow mode inside the ejector based on a supersonic ejector using CFD methods. They suggested that the RNG (renormalisation group) $k-\varepsilon$ model be applied to simulate a supersonic ejector [32]. Victor and Steven also verified the RNG $k-\varepsilon$ turbulence model as being able to simulate the flow characteristic based on a Coanda ejector. Therefore, in the present work, the RNG $k-\varepsilon$ double equation model [33] was applied to the annular slot ejector as follows.

$k$ equation:

$$
\frac{\partial(\rho k)}{\partial t}+\frac{\partial\left(\rho k u_{i}\right)}{\partial x_{i}}=\frac{\partial\left(\alpha_{k} \mu_{\mathrm{eff}}\left(\partial k / \partial x_{j}\right)\right)}{\partial x_{j}}+G_{k}-\rho \varepsilon
$$

where $\rho$ is the fluid density, $k$ is the turbulent kinetic energy, $t$ is the time, $\alpha_{k}$ is the turbulent Prandtl number of $k, \mu_{\mathrm{i}}$ and $\mu_{\mathrm{eff}}$ are the viscosity coefficients, $x_{i}$ and $x_{j}$ are coordinate vectors, $G_{k}$ represents the turbulent kinetic energy generated by the laminar velocity gradient, and $\varepsilon$ is the dissipation rate.

$\varepsilon$ equation: 


$$
\begin{aligned}
\frac{\partial(\rho \varepsilon)}{\partial t}+\frac{\partial\left(\rho \varepsilon u_{i}\right)}{\partial x_{i}}= & \frac{\partial\left(\alpha_{\varepsilon} \mu_{e f f} \partial \varepsilon / \partial x_{j}\right)}{\partial x_{j}} \\
& +C_{1 \varepsilon} * \frac{\varepsilon}{k} G_{k}-C_{2 \varepsilon} \rho \frac{\varepsilon^{2}}{k} \\
C_{1 \varepsilon} *= & C_{1 \varepsilon}-\frac{\eta\left(1-\left(\eta / \eta_{0}\right)\right)}{1+\beta \eta^{3}}, \\
\eta= & \sqrt{2 E_{i j}} \frac{k}{\varepsilon}, \\
E_{i j}= & \frac{1}{2}\left(\frac{\partial u_{i}}{\partial x_{j}}+\frac{\partial u_{j}}{\partial x_{i}}\right),
\end{aligned}
$$

where $C_{1 \varepsilon}$ and $C_{2 \varepsilon}$ are constants, indicating the effect of buoyancy on the dissipation rate, and $\alpha_{\varepsilon}$ is the turbulent Prandtl number of $\varepsilon$. The values of the coefficients in the formula are as follows:

$$
\begin{aligned}
\alpha_{k} & =\alpha_{\varepsilon}=1.39, \\
\beta & =0.012, \\
\eta_{0} & =4.377, \\
C_{\mu} & =0.0845, \\
C_{1 \varepsilon} & =1.42, \\
C_{2 \varepsilon} & =1.68 .
\end{aligned}
$$

\subsection{Modelling and Meshing for an Annular Slot Ejector.} An annular slot ejector is usually an axisymmetric structure, which includes eight parts (Figure 2). Moreover, the 3D model can be simplified to a $2 \mathrm{D}$ model according to the flow characteristic. The mainstream gas flows at high speed along the wall after passing through the nozzle clearance. Meanwhile, a secondary flow will be induced into the mixing chamber. Thereafter, the mixing gas flows outward through the diffusion chamber; however, the velocity near the wall is different from that near the centreline due to the Coanda effect, which causes a physical gradient inside the ejector; therefore, the corresponding mesh mapped using gridgenerating software (ICEM) is encrypted, to guarantee the accuracy of the numerical simulation results. All elements are quadrilaterals with about 200,000 in each mesh. The mesh size near the wall boundary is $0.1 \mathrm{~mm}$ and it gradually increases to $1 \mathrm{~mm}$ (Figure 3(a)). The independence of the grid has been analysed by obtaining a resulting mesh size of $0.75 \mathrm{~mm}$. Three meshes (a coarse grid, medium grid, and fine grid) were used to evaluate grid size-independence; the three grid sizes tested were $0.75 \mathrm{~mm}, 1 \mathrm{~mm}$, and $1.25 \mathrm{~mm}$, respectively. Figure 3(b) shows that secondary mass flow increases first and then decreases as the primary pressure increases from $0.3 \mathrm{MPa}$ to $0.7 \mathrm{MPa}$; the difference in secondary mass flow for the fine and medium grid is observed to be slight, but there was some improvement compared with the case modelled using a grid size of $1 \mathrm{~mm}$. Therefore, the simulation model with a $1-\mathrm{mm}$ grid was selected to reduce the computational time and ensure accuracy in subsequent simulations.

2.3. Boundary Conditions. The operating fluid is a compressed gas, and the ejector fluid is from the surrounding air. In the present work, both fluids are treated as ideal gases, as carried out by other scholars [29, 34]. An implicit solution method and a hybrid initialisation method were applied to the flow calculation of compressible gases. Both the highpressure inlet and the low-pressure inlet of the annular slot ejector were set as a pressure inlet boundary condition, and the mixed fluid outlet is configured as a pressure outlet. Other boundaries are wall surfaces, and the insulation between the wall and the environment is assumed to be adiabatic, nonpermeable, and nonslip boundaries [35] (Table 1).

\section{Analysis of Factors Affecting Ejector Performance}

3.1. Influence of Mixing Chamber Length. Figure 4 shows that the entrainment ratio raises first and then decreases as the length of the mixing chamber increases from $10 \mathrm{~mm}$ to $100 \mathrm{~mm}$, and the corresponding maximum entrainment ratio is obtained when the mixing chamber length is $40 \mathrm{~mm}$. There is a certain buffer developed during the mixing process of primary and secondary flows. More importantly, when the mixing chamber is short, mainstream gas does not transmit momentum to the ejector fluid well, resulting in the insufficient mixing of the two fluids; moreover, when the length of the mixing chamber is too large, the impact loss of the fluid increases. Figure 5 indicates that the distribution of the flow velocity under different primary pressures include 0.3 $\mathrm{MPa}, 0.4 \mathrm{MPa}, 0.5 \mathrm{MPa}, 0.6 \mathrm{MPa}$, and $0.7 \mathrm{MPa}$, respectively. It is seen that the flow velocity away from the wall shows a certain gradient, and there is good turbulent mixing at the interface between the primary gas and the secondary gas [36]. In addition, for different primary pressures, the flow velocity near the wall is always much larger than that near the central axis, which corresponds to the main features of the Coanda effect [37].

3.2. Influence of Diffusion Chamber Length. Figure 6 shows the relationship between diffusion chamber length and entrainment ratio when the mainstream pressure is $0.3 \mathrm{MPa}$; the entrainment ratio continuously increases as the diffusion chamber length increases from $120 \mathrm{~mm}$ to $400 \mathrm{~mm}$. This can be explained by their different physical flow processes; the flow of the mixed fluid in the diffusion chamber is a process in which static pressure recovery quickly, and the rapid decline of velocity and the diffusion area increases with a certain gradient during the process (Figure 7). The highpressure jet layer is wider when the diffusion chamber length is $400 \mathrm{~mm}$ than at other sizes, suggesting good turbulent mixing. Moreover, the diffusion area contributes to the amount of induced air during the process, and it may be necessary to use more compressed gas to complete turbulent mixing with the induced fluid. 


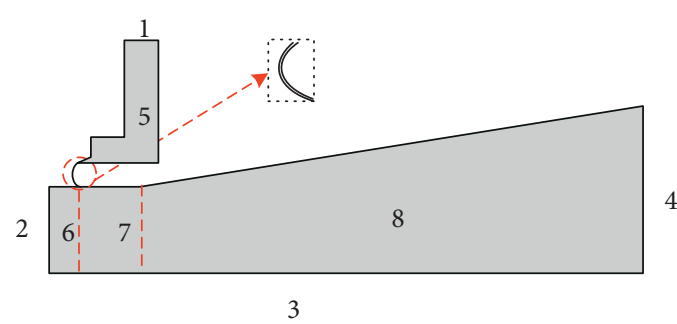

(a)

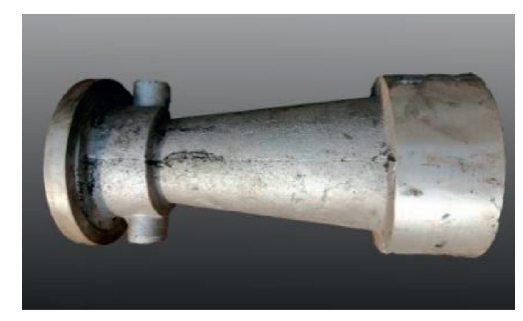

(b)

FIgURE 2: The annular slot ejector model. (a) Schematic diagram of annular slot ejector and (b) photograph of the physical model. 1. Highpressure inlet. 2. Secondary inlet. 3. Symmetry axis. 4. Outlet. 5. Storage room. 6. Suction. 7. Mixing chamber. 8. Diffusion chamber.

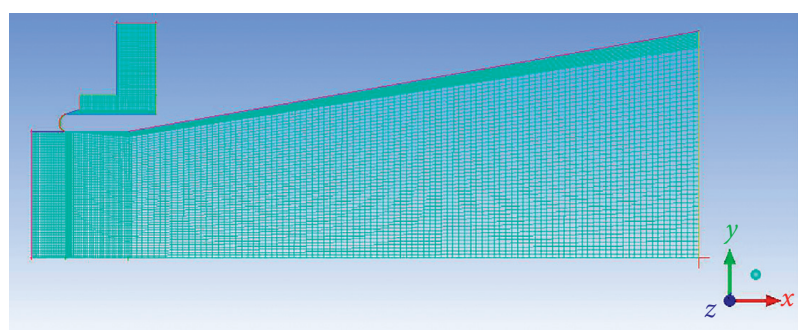

(a)

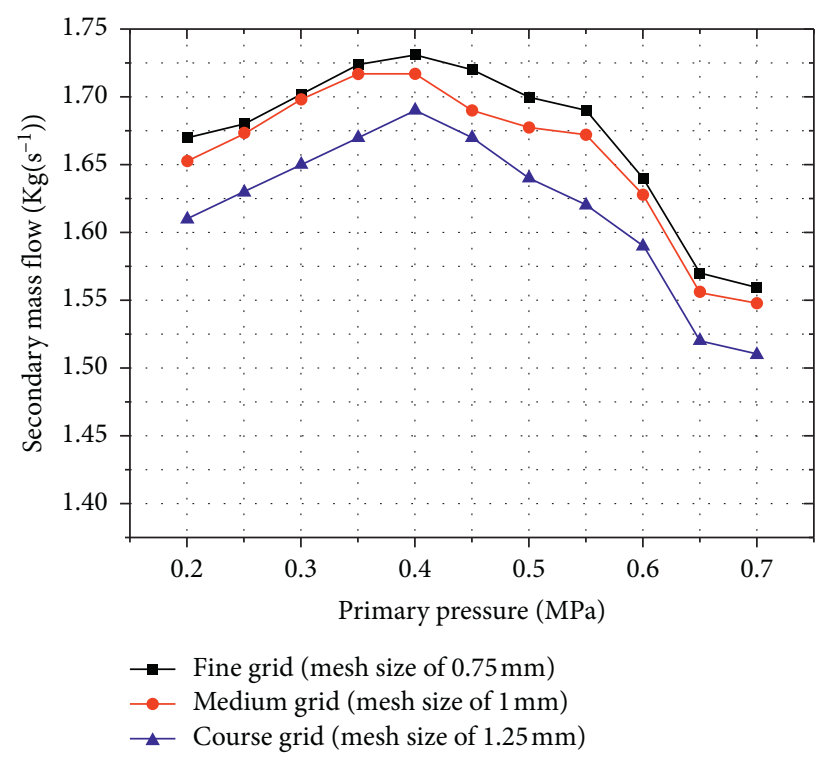

(b)

Figure 3: (a) Meshing for annular slot ejector and (b) secondary mass flow with different grid densities.

TABLE 1: Boundary conditions.

\begin{tabular}{lcccc}
\hline Location & Pressure & Turbulence intensity & Hydraulic diameter $(\mathrm{m})$ & Total temperature $(\mathrm{K})$ \\
\hline Primary inlet & $3-7 \times 10^{5} \mathrm{~Pa}$ & 1 & 0.025 & 0.08 \\
Secondary inlet & $1 \times 10^{5} \mathrm{~Pa}$ & 1 & 0.16 & 300 \\
Outlet & $1 \times 10^{5} \mathrm{~Pa}$ & 5 & & \\
\hline
\end{tabular}

3.3. Influence of the Diffusion Chamber Angle. Figure 8 indicates that the entrainment ratio decreases as the angle of the diffusing chamber increases from $6^{\circ}$ to $16^{\circ}$; the highpressure jet is not separated from the wall surface as the angle increases due to the Coanda effect. Nevertheless, the high-pressure jet layer becomes thinner and the corresponding mixed boundary layer moves towards the wall because the adsorption capacity decreased. Moreover, due to the increase of the diffusion area, the amount of induced air rises (in relative terms), and the traction force on the induced fluid is reduced, thereby causing the entrainment ratio to decrease at the same primary pressure. Figure 9 indicates the relationship between the velocity and the angle of the diffusing chamber; the flow velocity decreases upon widening of the diffusion chamber angle. Besides, when the diffusion angle is large enough, a local counterflow zone appears near the inlet section of the diffusion chamber and the surrounding countercurrent zone still contains higherenergy fluid, which may cause energy loss. Therefore, the attenuation of the jet flow may be greater, further resulting in a lower entrainment ratio.

3.4. Influence of Throat Diameter. Figure 10 shows that the mass flow both primary and secondary gas increases with the increase of the throat diameter, and the growth rate of the 


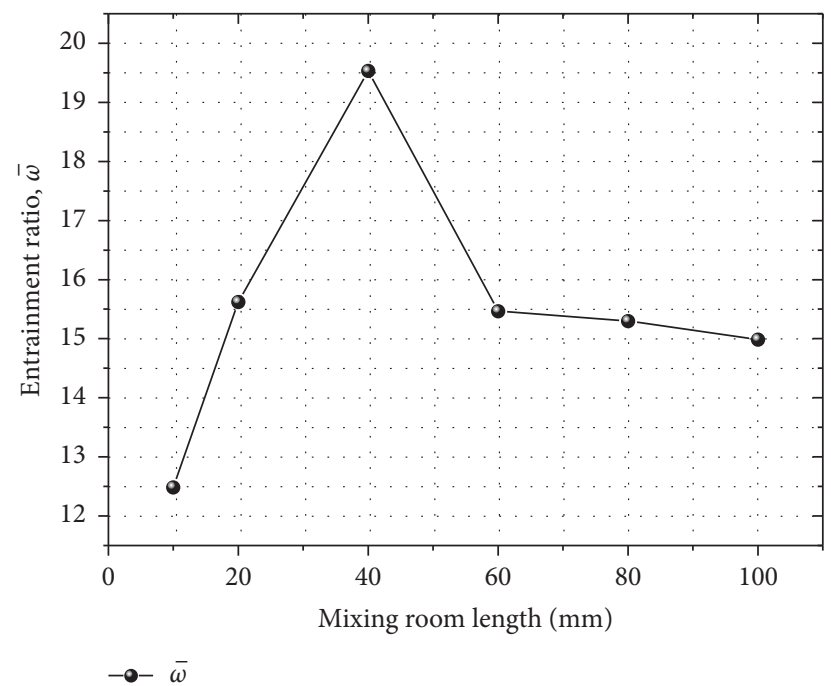

Figure 4: Mixing chamber length $v$ entrainment ratio.

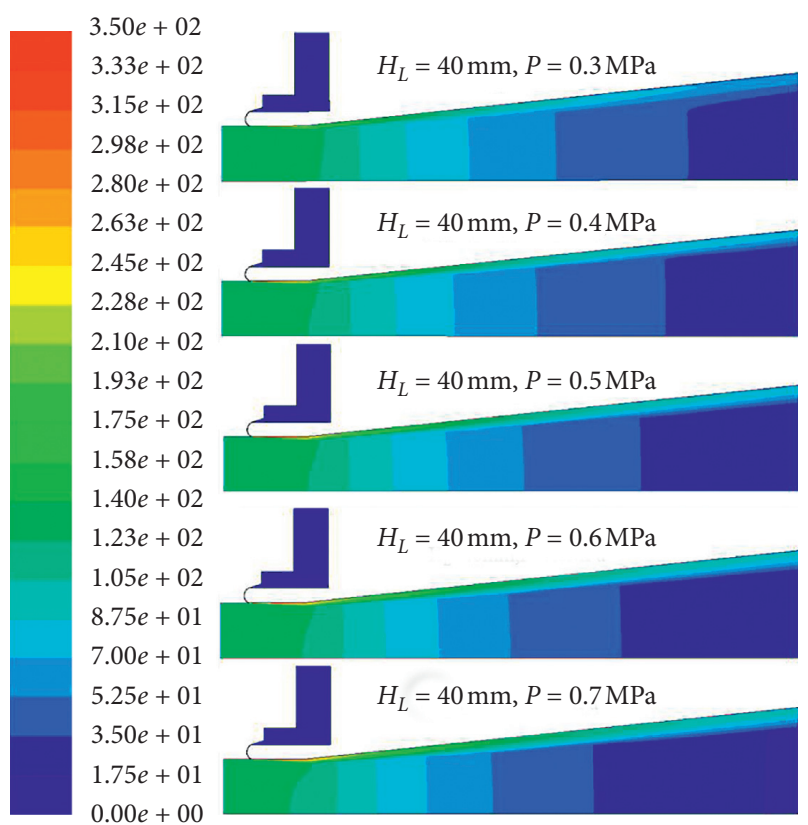

FIGURE 5: Velocity contours inside the ejector at different primary pressures.

secondary mass flow rate is significantly higher than that of the primary flow. This can be explained by considering that the aspect ratio both primary and secondary flows increase as throat diameter rises, inducing more air, and resulting in a greater mass flow rate. Figure 11 shows that the entrainment ratio decreases significantly as the primary pressure increases from $0.3 \mathrm{MPa}$ to $0.7 \mathrm{MPa}$, which can be explained by the fact that the larger primary pressure is, the more mass flow is available for primary flow, resulting in a lower entrainment ratio; however, the entrainment ratio shows a complicated trend in behaviour upon variation of the throat diameter, which may lie in the complex physical processes including turbulent flow mixing in both primary and

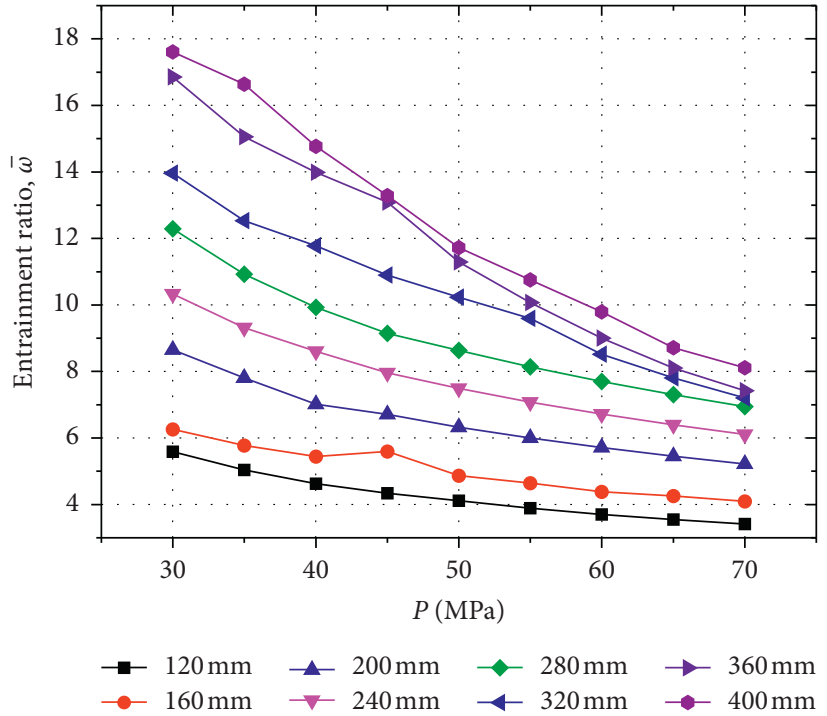

FIGURE 6: Diffusion chamber length $v$ entrainment ratio.

secondary flows. In addition, as the aspect ratio increases, the more shearing force will be needed to drag the secondary flow in. Thereafter, the primary and secondary flows enter the mixing chamber to achieve static pressure matching, completing the mixing process of the two flows inside the mixing chamber, reducing the impact loss between the two fluids. At the same time, as the throat diameter increases from $60 \mathrm{~mm}$ to $160 \mathrm{~mm}$, more air (by volume) is induced into the ejector. In previous studies, Kim et al. analysed the influence of throat diameter on the ejector coefficient based on a Coanda ejector [28]. Their results showed that the velocity decreases when the throat diameter increases from $35 \mathrm{~mm}$ to $50 \mathrm{~mm}$; however, the higher velocity does not mean a larger mass flow due to the decline in diameter resulting in a lower flow. In the present work, the mixing entrainment ratio is obtained when the throat diameter is $160 \mathrm{~mm}$ under different primary pressures. 


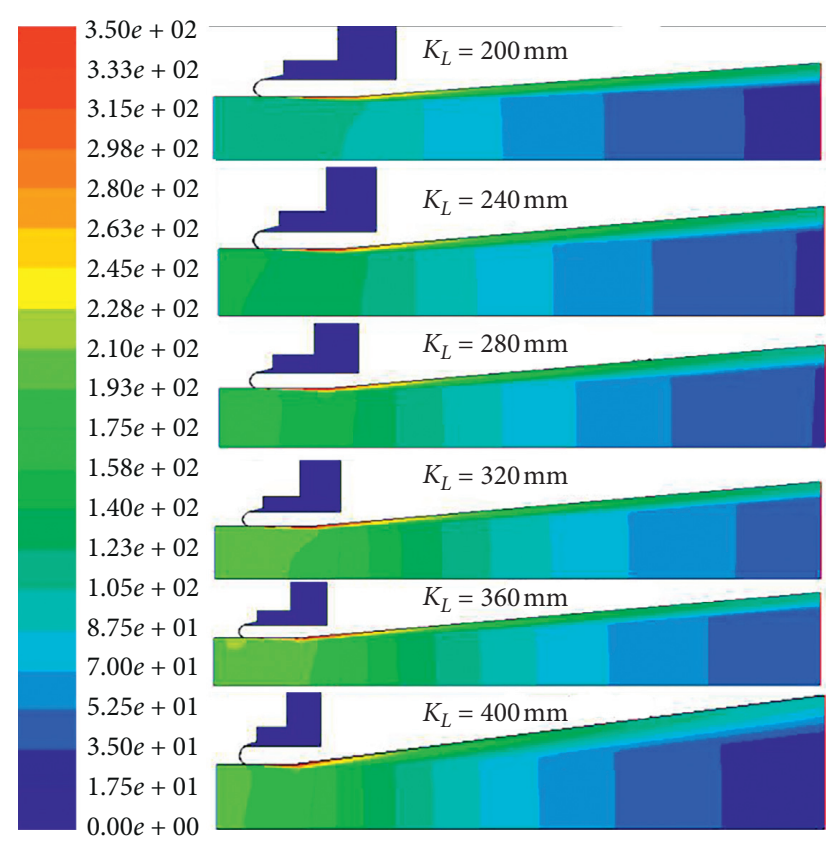

FIGURE 7: Velocity contours inside the ejector for different diffusion chamber lengths.

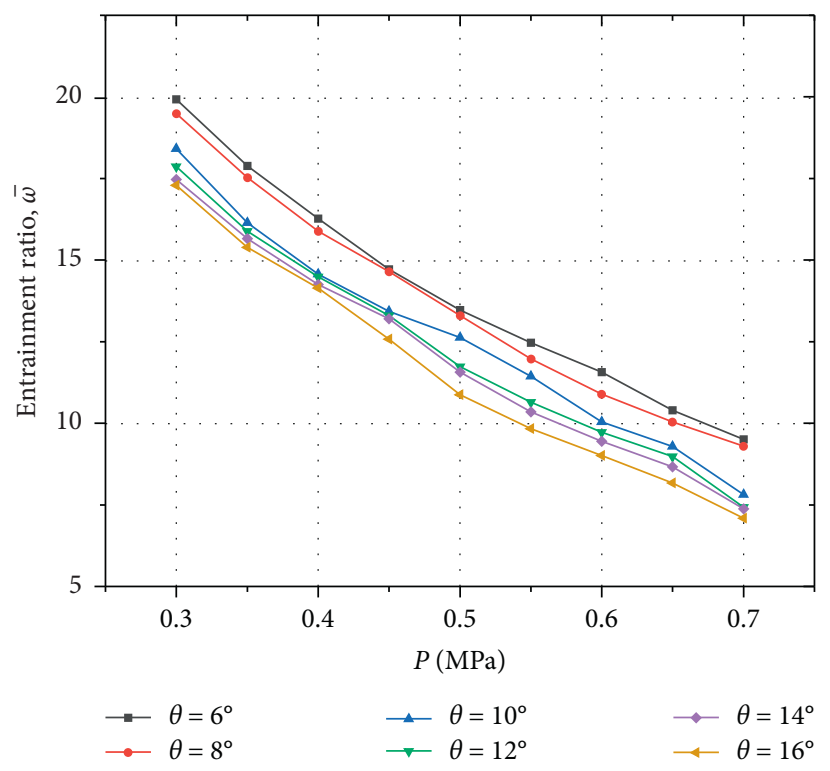

FIGURE 8: Diffusion chamber angle $v$ entrainment ratio.

3.5. Influence of Nozzle Clearance. Figure 12 shows that the entrainment ratio gradually decreases as the primary pressure increases from $0.3 \mathrm{MPa}$ to $0.7 \mathrm{MPa}$. When the mainstream pressure is fixed, the entrainment ratio decreases continuously as the nozzle clearance increases from $0.1 \mathrm{~mm}$ to $0.5 \mathrm{~mm}$; a similar trend was obtained elsewhere [30]. It also can be seen that when the primary pressure is $0.3 \mathrm{MPa}$, the entrainment ratio increases by $42.3 \%$ as the nozzle clearance increases from $0.1 \mathrm{~mm}$ to $0.15 \mathrm{~mm}$, and the entrainment ratio rises by $85.9 \%$ when the nozzle clearance increases from $0.1 \mathrm{~mm}$ to $0.5 \mathrm{~mm}$. Therefore, the nozzle

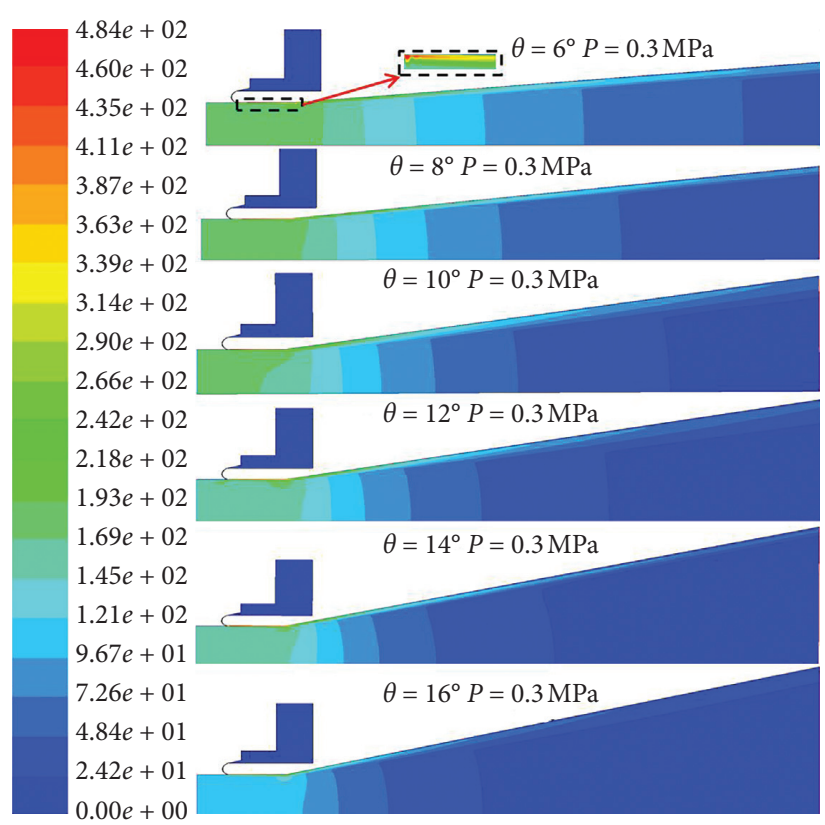

FIGURE 9: Velocity contours inside the ejector for different angles.

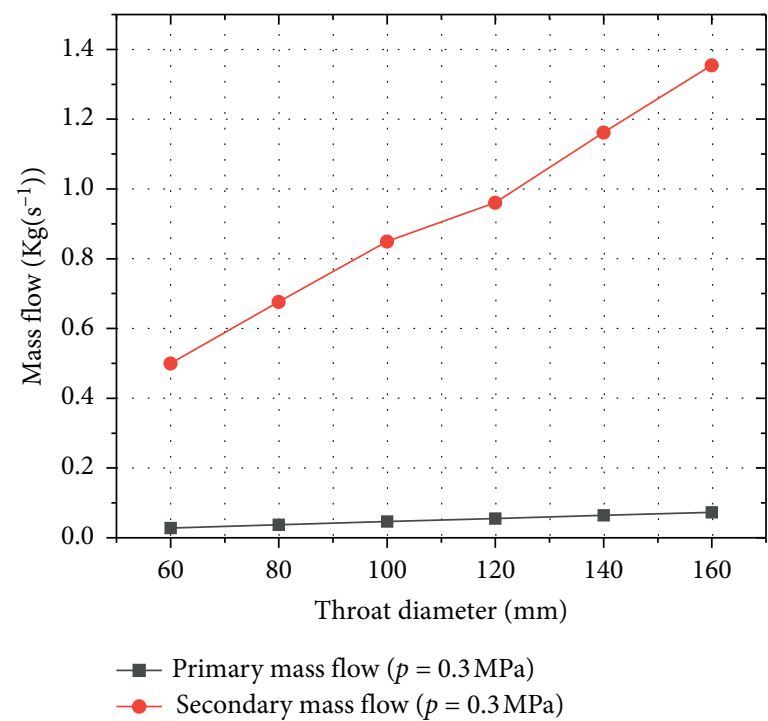

Figure 10: Throat diameter of diffusion chamber $v$ mass flow.

clearance affects the performance of the annular slot ejector to a significant extent.

3.6. Multifactor Analysis of Ejector Performance Employing the Grey Relational Analysis Method. The above analysis shows the influence of the geometry on the entrainment ratio when one parameter changes but other parameters are fixed. Nozzle clearance is the more important parameter; nevertheless, the importance of the other parameters in terms of their influence on ejector performance is unclear; therefore, it is necessary to analyse ejector performance when the five geometric parameters are varied simultaneously. The grey relational analysis method involves the analysis of an 


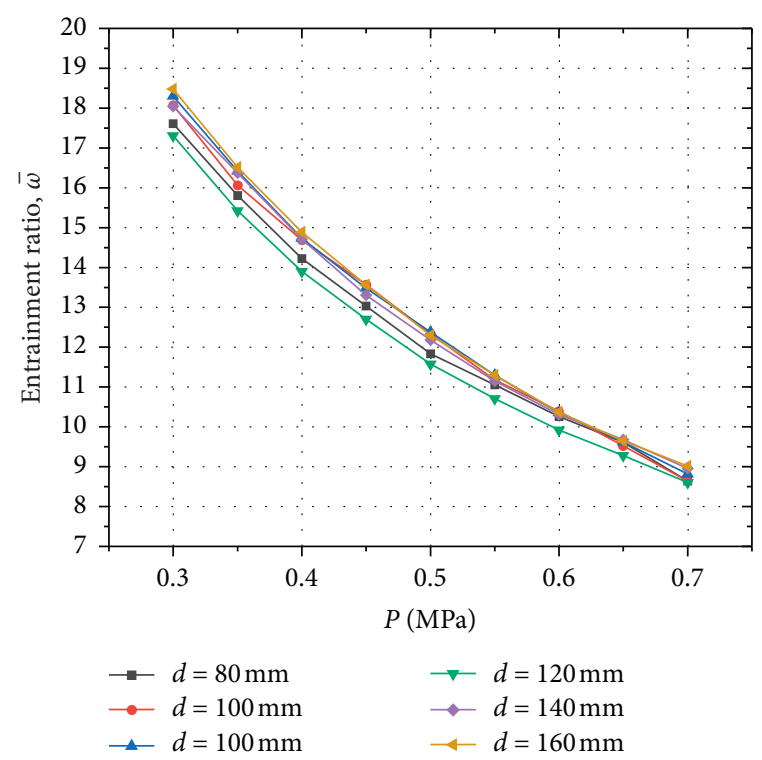

FIgURE 11: Throat diameter $v$ entrainment ratio.

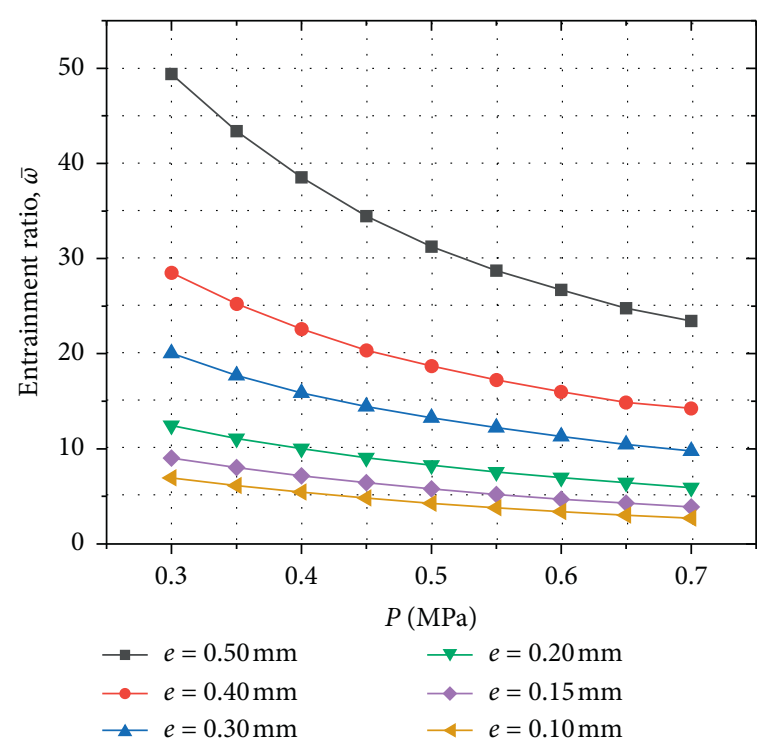

Figure 12: Nozzle clearance $v$ entrainment ratio.

abstract system or phenomenon, which makes up for the deficiencies in systematic analysis using mathematical statistical methods. It is also applicable to any number of samples and works irrespective of a parametric distribution being known a priori. Ju-Long [38] proposed a theoretical model for analysing the correlation between samples:

$$
\gamma_{0 i}(k)=\frac{\min _{i} \min _{k}\left|\left(X_{0}(k)-X_{i}(k)\right)\right|+\xi \max _{i} \max _{k}\left|\left(X_{0}(k)-X_{i}(k)\right)\right|}{\left|\left(X_{0}(k)-X_{i}(k)\right)\right|+\xi \max _{i} \max _{k}\left|\left(X_{0}(k)-X_{i}(k)\right)\right|},
$$

where $X_{i}(k)$ is observed data on sequence $k, X_{0}(k)$ reflects the behaviour of the system characteristics, $k$ can also represent the time serial number and the index number, $\gamma_{0 i}(k)$ is the grey correlation of $X_{i}$ and $X_{0}$, and $\xi$ is the resolution factor.
In the present work, the grey relational analysis method was applied to study ejector performance without fixed geometric parameters. A set of numerical simulations were conducted to investigate the ejector performance under constant pressure (Table 2). Taking the first set of parameters from Table 2 as a reference sequence, the six sets of parameters were normalised and initialised (Table 3). Finally, the sensitivity of each structure parameter to the entrainment ratio was obtained (Table 4) which can be sorted as follows: nozzle clearance $(e)>$ diffusion chamber angle $(\theta)>$ mixing chamber length $\left(H_{L}\right)>$ throat diameter $(d)>$ diffusion chamber length $\left(K_{L}\right)$.

\section{Experimental Optimisation Model of an Annular Slot Ejector}

4.1. Experiments. Although a series of numerical calculations were conducted to optimise the ejector structure, the optimised model still needs further experimental verification. The optimised model and dimensions were obtained based on the aforementioned simulated results, and the comparison between the original model and the optimised model is shown in Figure 13 and Table 5 (where $D_{\text {main }}$ is the diameter of the primary inlet, $D_{\mathrm{sec}}$ is the diameter of the secondary inlet, and $D_{\text {out }}$ is the diameter of the outlet).

In this experiment, the logarithmic linear measurement method [39] was used to measure the average dynamic pressure in the ejector inlet section, and then the average velocity and mass flow of the ejector inlet section were obtained. The experiments were conducted in a relatively closed indoor environment (Figure 14). An air compressor was adopted to provide mainstream gas flow under different pressures and to act as a buffer airflow. The gas collection tank was used to supply a continuous flow of compressed gas. Moreover, the compressed air was dried before entering the ejector. The pressure transmitter measured the mainstream pressure, and the gas turbine flowmeter was used to measure the primary mass flow and a Pitot tube was utilised to measure the dynamic pressure at each measurement point on the cross-section of the ejector exit and to obtain the average flow velocity and the total mass flow. These three parameters were transmitted through a sensor to a computer program for simultaneous counting with a pulse counter.

4.2. Analysis of Experimental Results. Nine groups of experimental tests present a set of parameters for ejector performance at different primary pressures (Table 6); the primary mass flow $\left(G_{1}\right)$ rises continuously while the secondary mass flow $\left(G_{2}\right)$ tends to first increase, then decrease as the primary pressure increases from $0.3 \mathrm{MPa}$ to $0.7 \mathrm{MPa}$. Moreover, the dynamic pressure and velocity in the ejector exit show the same trend as the secondary flow; this can be explained by considering that the excessive primary pressure developed poor turbulent mixing, forming a local vortex, thereby leading to a decrease in the secondary mass flow rate. The entrainment ratio ( $\omega)$ decreases continuously with increasing primary pressure (Figure 15). Moreover, there is good consistency found by comparing simulated results and 
TABLe 2: Preliminary calculated entrainment ratios.

\begin{tabular}{llcccc}
\hline$H_{L}$ & $K_{L}$ & $\theta$ & $d$ & $e$ & Entrainment ratio \\
\hline 10 & 200 & 6 & 80 & 0.1 & 37.431 \\
20 & 240 & 8 & 100 & 0.15 & 20.756 \\
40 & 280 & 10 & 120 & 0.2 & 21.461 \\
60 & 320 & 12 & 140 & 0.3 & 14.286 \\
80 & 14 & 160 & 0.4 & 10.987 \\
100 & 360 & 16 & 180 & 0.5 & 9.301 \\
\hline
\end{tabular}

TABle 3: Pretreatment results.

\begin{tabular}{lccccc}
\hline$X_{0}$ & $X_{1}$ & $X_{2}$ & $X_{3}$ & $X_{4}$ & $X_{5}$ \\
\hline 1 & 1 & 1 & 1 & 1 & 0.8 \\
0.554514 & 0.5 & 0.833333 & 0.75 & 0.666667 & 0.666667 \\
0.573346 & 0.25 & 0.714286 & 0.6 & 0.571429 & 0.5 \\
0.381654 & 0.166667 & 0.625 & 0.5 & 0.428571 & 0.333333 \\
0.293524 & 0.125 & 0.555556 & 0.375 & 0.25 \\
0.248495 & 0.1 & 0.5 & & 0.2 \\
\hline
\end{tabular}

TABLE 4: Relevance ranking results.

\begin{tabular}{lcc}
\hline Structural parameter & Correlation & Rank \\
\hline Mixing chamber length & 0.586878 & 3 \\
Diffusion chamber length & 0.51222 & 5 \\
Diffusion chamber angle & 0.66573 & 2 \\
Throat diameter & 0.563725 & 4 \\
Nozzle clearance & 0.767563 & 1 \\
\hline
\end{tabular}

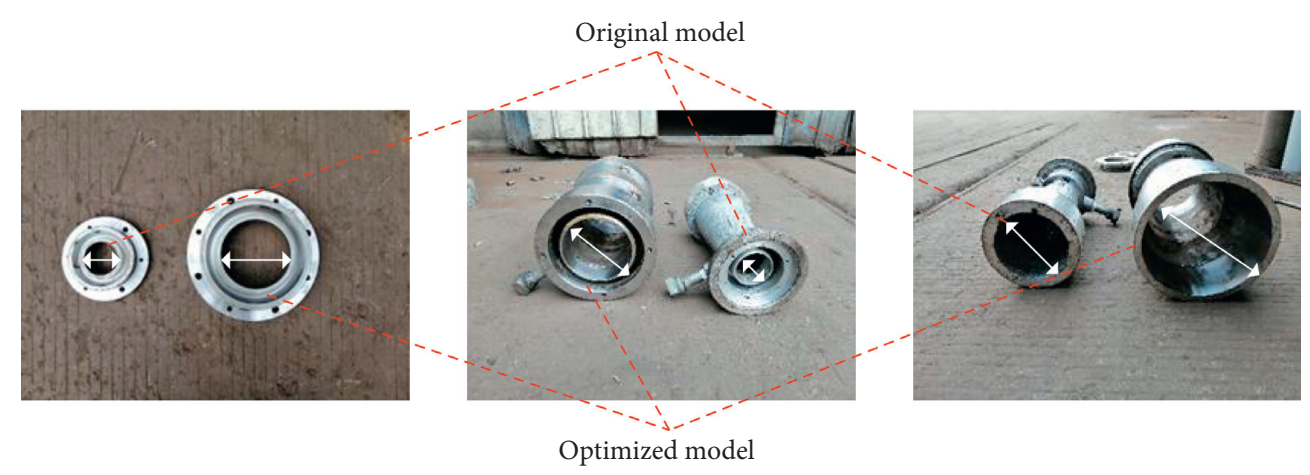

FIGURE 13: Comparison of annular ejector models. (a) Gland, (b) secondary inlet, and (c) outlet.

TABLE 5: Improved model dimensions for an annular slot ejector.

\begin{tabular}{lcc}
\hline Structural parameter & Origin size $(\mathrm{mm})$ & Optimised size $(\mathrm{mm})$ \\
\hline$D_{\text {main }}$ & 25 & 25 \\
$D_{\text {sec }}$ & 80 & 160 \\
$D_{\text {out }}$ & 145 & 290 \\
$d$ & 80 & 160 \\
$e$ & 0.3 & 0.1 \\
$\theta$ & 60 & $6^{\circ}$ \\
$H_{L}$ & 20 & 40 \\
$K_{L}$ & 300 & 400 \\
\hline
\end{tabular}




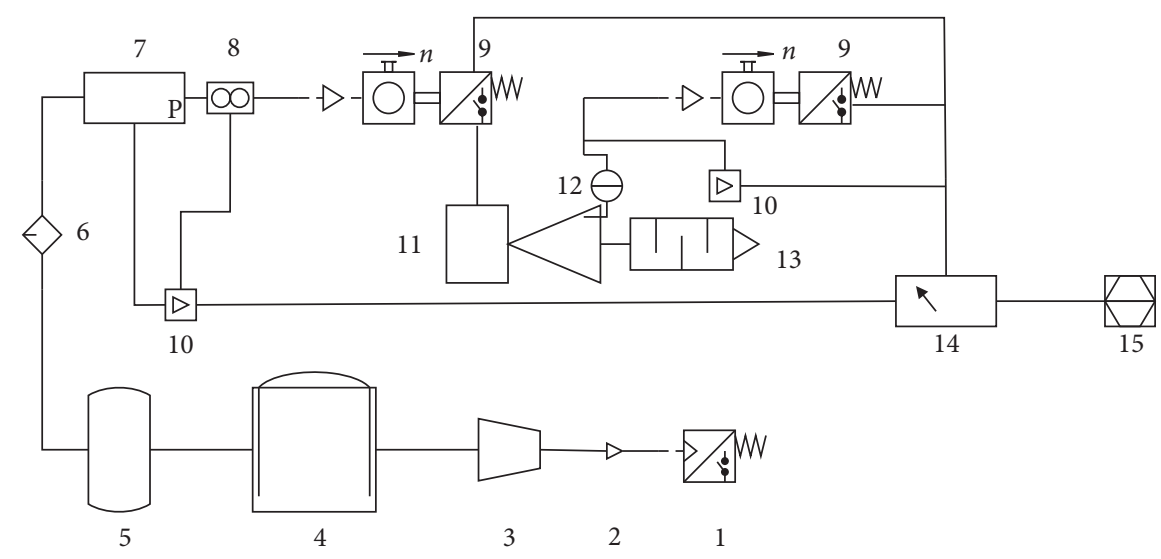

FIGURE 14: Schematic diagram of the experimental flow regime in the annular ejector. 1. Power switch. 2. Starting device. 3. Air compressor. 4. Gas tank. 5. Buffer gas tank. 6. Desiccator. 7. Pressure transmitters. 8. Gas vortex flowmeter. 9. Pulse counter. 10. Sensor. 11. Annular slot ejector. 12. L-type pitot tube and differential manometer. 13. Silencer. 14. Monitor. 15. Computer.

TABLe 6: Experimental results.

\begin{tabular}{|c|c|c|c|c|c|}
\hline$P_{1}(\mathrm{MPa})$ & $G_{1}(\mathrm{~kg} / \mathrm{s})$ & $G_{2}(\mathrm{~kg} / \mathrm{s})$ & $\omega$ & $P(\mathrm{~Pa})$ & $V\left(\mathrm{~m}^{3} / \mathrm{s}\right)$ \\
\hline 0.30 & 0.0234 & 0.991 & 41.367 & 107.28 & 13.154 \\
\hline 0.35 & 0.0276 & 1.061 & 37.428 & 122.97 & 14.083 \\
\hline 0.40 & 0.0298 & 1.097 & 35.805 & 131.45 & 14.561 \\
\hline 0.45 & 0.0341 & 1.220 & 34.780 & 162.59 & 16.194 \\
\hline 0.50 & 0.0375 & 1.291 & 33.440 & 182.06 & 17.136 \\
\hline 0.55 & 0.0409 & 1.257 & 31.678 & 172.60 & 16.685 \\
\hline 0.60 & 0.0435 & 1.248 & 27.678 & 170.15 & 16.566 \\
\hline 0.65 & 0.0463 & 1.209 & 25.118 & 159.67 & 16.048 \\
\hline 0.70 & 0.0576 & 1.164 & 19.201 & 148.01 & 15.451 \\
\hline
\end{tabular}

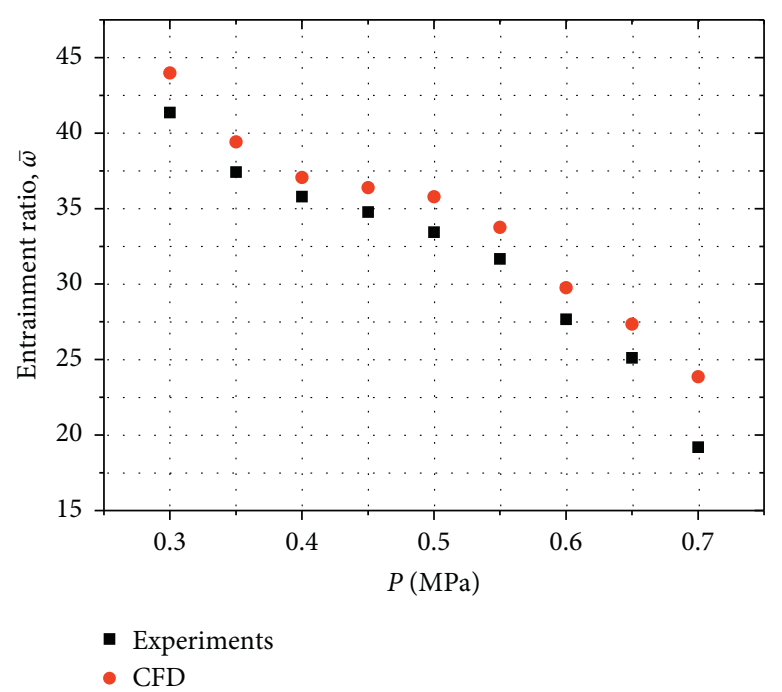

FIGURE 15: Comparison of experimental and numerical simulation results.

experimental data, which also indicates that the RNG- $k-\varepsilon$ turbulent transport mode can simulate the flow characteristics of the gas in the ejector. Besides, it could be seen that numerical simulation results are always slightly higher than experimental test values, which can be explained using the Boussinesq hypothesis, ensure solution closure, and the working flow was set to that of an ideal gas.

\section{Conclusion}

To investigate the annular slot ejector performance, a twodimensional ejector structure model was constructed employing Fluent 15.0. Five factors $\left(e, \theta, H_{L}, d\right.$, and $\left.K_{L}\right)$ were selected to analyse ejector performance; while one parameter was changed, the others were fixed. Then, the grey correlation analysis was used to study ejector performance when the five structural parameters were changed simultaneously, and the correlation of the performance parameters affecting the annular ejector was obtained. Finally, the optimised model was verified by comparing the numerical results with experimental data. The main conclusions were as follows.

$@$ first rises, then falls when increasing $H_{L}$ from $10 \mathrm{~mm}$ to $100 \mathrm{~mm}$, and when $H_{L}$ was $40 \mathrm{~mm}$, the maximum value of $₫$ was obtained; there is a nonmonotonic trend seen when $D$ rises from $80 \mathrm{~mm}$ to $160 \mathrm{~mm}$. When $\theta$ increases from $6^{\circ}$ to $16^{\circ}$, there is a continuously decreasing trend; however, when varying $K_{L}$ (from $120 \mathrm{~mm}$ to $400 \mathrm{~mm}$ ) and $e$ (from $0.1 \mathrm{~mm}$ to $0.5 \mathrm{~mm}$ ), $₫$ increased at all times. Therefore, the dimensions of the optimised model were as follows: $H_{L}=40 \mathrm{~mm}, K_{L}=400 \mathrm{~mm}, \theta=6^{\circ}, d=160 \mathrm{~mm}$, and $e=0.5 \mathrm{~mm}$. The grey correlation between the five factors and the ejector performance was obtained as follows: $e>\theta>H_{L}>d>K_{L}$; therefore, the nozzle clearance is the most important parameter among the five factors affecting ejector performance. Nine groups of primary pressure conditions (from $0.3 \mathrm{MPa}$ to $0.7 \mathrm{MPa}$ ) were 
analysed using a set of experiments based on the optimised model to verify the accuracy of the simulated entrainment ratio; the corresponding results indicated that the two methods were consistent. Besides, the feasibility of the use of the RNG- $k-\varepsilon$ turbulent transport mode was verified in simulating the flow characteristics of the gas in the ejector.

\section{Data Availability}

The data used to support the findings of this study are included within the manuscript.

\section{Conflicts of Interest}

The authors declare that they have no conflicts of interest.

\section{Acknowledgments}

This work was supported by the National Natural Science Foundation of China (Grant nos. 51974232 and 51574193) and Fundamental Research Funds of Shaanxi Province, China (Grant no. 2017JM5039).

\section{References}

[1] Y. Han, L. Guo, X. D. Wang, and A. C. Y. Yuen, "A steam ejector refrigeration system powered by engine combustion waste heat: part 1 . characterization of the internal flow structure," Applied Sciences, vol. 9, no. 20, p. 4275, 2019.

[2] Y. Han, X. D. Wang, L. Guo, and A. Chun, "A steam ejector refrigeration system powered by engine combustion waste heat: part 2. understanding the nature of the shock wave structure," Applied Sciences, vol. 9, no. 20, 2019.

[3] Y. Han, X. Wang, A. C. Y. Yuen et al., "Characterization of choking flow behaviors inside steam ejectors based on the ejector refrigeration system," International Journal of Refrigeration, vol. 113, pp. 296-307, 2020.

[4] Y. Wu, H. Zhao, C. Zhang, L. Wang, and J. Han, "Optimization analysis of structure parameters of steam ejector based on CFD and orthogonal test," Energy, vol. 151, pp. 79-93, 2018.

[5] S. Da-Wen and W. E. Ian, "Recent developments in the design theories and applications of ejectors-a review," Fuel \& Energy Abstracts, vol. 36, no. 5, pp. 361-370, 1995.

[6] D. J. Pieiro, "Henry Marie Coanda and the "Coanda effect"” Revista Portuguesa De Pneumologia, vol. 80, no. 1, p. 4, 2010.

[7] C. Ionică, D. Sorin, and G. Virgil, "Theoretical approaches regarding the gasodynamic phenomena in asymmetric flows," Advanced Material Research, vol. 1128, pp. 364-371, 2015.

[8] C. Smith, "On some recent applications of the Coanda effect to acoustics," The Journal of the Acoustical Society of America, vol. 128, no. 4, p. 16, 2010.

[9] D. G. Gregory-Smith and A. R. Gilchrist, "The compressible Coanda wall jet--an experimental study of jet structure and breakaway," International Journal of Heat and Fluid Flow, vol. 8, no. 2, p. 9, 1987.

[10] S. Jain, Shashi, and S. Kumar, "Numerical studies on evaluation of smoke control system of underground metro rail transport system in India having jet injection system: a case study," Building Simulation, vol. 4, no. 3, pp. 205-216, 2011.

[11] I. Cîrciu and S. Dinea, Review of the Air Force Academy, "Henri Coandă" Air Force Academy, Braşov Romania, 2010.
[12] A. Perrig, F. Avellan, J.-L. Kueny, M. Farhat, and E. Parkinson, "Flow in a pelton turbine bucket: numerical and experimental investigations," Journal of Fluids Engineering, vol. 128, no. 2, pp. 350-358, 2006.

[13] E. Natarajan and N. O. Onubogu, "Application of Coanda effect in robots-a review," Mechanical Engineering and Technology, vol. 125, pp. 411-418, 2012.

[14] L. Guoqi, H. Yongjun, and Y. Yingzi, "Influence of Coanda surface curvature on performance of bladeless fan," Journal of Thermal Science, vol. 23, no. 5, p. 10, 2014.

[15] L. Dong-Won, H. Jae-Gun, K. Young-Doo, and K. Soon-Bum, "A study on the air knife flow with Coanda effect," Journal of Mechanical Science and Technology, vol. 21, no. 12, p. 7, 2007.

[16] D. Alexandru, F. Frunzulica, and C. I. Tudor, Mathematical Modelling and Numerical Investigations on the Coanda Effect, Nonlinearity, Bifurcation and Chaos-Theory and Applications, Intech, London, UK, 2012.

[17] D. Alexandru, F. Frunzulica, F. Frunzulica, and T. Ionescu, "Coanda effect on the flows through ejectors and channels," Scientific Research and Education in the Air Force, vol. 20, pp. 161-174, 2018.

[18] A. Dumitrache, F. Frunzulica, and O. Preotu, "Flow analysis in various ejectors configurations," in Proceedings of the 2017 Fourth International Conference on Mathematics and Computers in Sciences and in Industry, p. 7, Corfu, Greece, August 2017.

[19] A. Dumitrache, "Numerical investigation of the flow in a Coanda ejector," in Proceedings of the 4th European Conference For Aerospace Sciences (EUCASS), p. 12, Saint Petersburg, Russia, July 2011.

[20] V. Rajalakshmi, K. Kavitha, and D. Lavanya, "Design and optimization of single head planar Coanda gripper," Advances in Natural and Applied Sciences, vol. 11, no. 4, p. 8, 2017.

[21] V. Benche and V. Benche, "Transient proceses for ventejectors assisted by Coanda effect," in Proceedimgs of the 6th International Conference on Hydraulic Machinery and $\mathrm{Hy}$ drodynamics Timisoara, p. 6, Timişoara, Romania, October 2004.

[22] T.-H. Kim, A Study on the Characteristics of Coanda Nozzle Flow, Saga University, Saga, Japan, 2007.

[23] P. M. Weston, V. Sharifi, and J. Swithenbank, "Destruction of tar in a novel Coandă tar cracking system," Energy \& Fuels, vol. 28, no. 2, pp. 1059-1065, 2014.

[24] H. C. Yang, "Horizontal two-phase jet behavior with an annular nozzle ejector in the water tank," Journal of Visualization, vol. 18, no. 2, pp. 359-367, 2014.

[25] C. P. Lubert, "Some recent experimental results concerning turbulent Coanda wall jets," The Journal of the Acoustical Society of America, vol. 136, no. 4, p. 2137, 2015.

[26] A. Mohammad, An Experimental and Theretical Study of Coanda ejectors, Case Western Reverse University, Cleveland, OH, USA, 1993.

[27] V. Guerriero, "Numerical solutions of compressible flow mixing in Coanda ejectors," in Proceedings of the Eighth Symposium on Fluid Control, Measurement and Visualization, China Society of Theoretical and Applied Mechanics, Chengdu, ChinaChina Society of Theoretical and Applied Mechanics, Chengdu, China, August 2005.

[28] H. D. Kim, G. Rajesh, T. Setoguchi, and S. Matsuo, "Optimization study of a Coanda ejector," Journal of Thermal Science, vol. 15, no. 4, pp. 331-336, 2006.

[29] G. Rajesh, "A computational study of the gas flow in a Coanda ejector," in Proceedings of the Korean Society of Mechanical Engineers Conference, Busan, Korea, June 2005. 
[30] J. A. Sierra del Rio, J. G. Ardila Marin, S. Vélez Garcia, M. Londoño, and D. A. Hincapie Zuluaga, "Simulation analysis of a coanda-effect ejector using CFD," Tecciencia, vol. 12, no. 22, pp. 17-25, 2016.

[31] K. P. Lowry, R. Y Coley, D. L Miglioretti et al., "Effect of Coanda nozzle clearance on the flow characteristics of air amplifier," in Proceedings of the 2014 6th International Symposium on Fluid Machinery and Fluid Engineering, Wuhan, China, March 2014.

[32] H. Amel, F. Henry, and S. Leclaire, "CFD analysis of a supersonic air ejector. Part I: experimental validation of singlephase and two-phase operation," Applied Thermal Engineering, vol. 29, no. 8, p. 9, 2009.

[33] Y. Victor and A. O. Steven, "Renormalization group analysis of turbulence I. basic theory," Journal of Scientific Computing, vol. 1, no. 1, pp. 3-51, 1986.

[34] D. Valentín, A. Guardo-Zabaleta, and E. Egusquiza, "Use of Coandă nozzles for double glazed fac, ades forced ventilation," Energy \& Buildings, vol. 62, p. 10, 2013.

[35] A. Li, A. C. Y. Yuen, T. B. Y. Chen, and C. Wang, "Computational study of wet steam flow to optimize steam ejector efficiency for potential fire suppression application," Applied Sciences, vol. 9, no. 7, 2019.

[36] E. F. Schum, P. M. Bevilaqua, and S. V. Patankar, Computation of the Turbulent Mixing in Curved Ejectors, Rockwell International Corporation, Milwaukee, WI, USA, 1980.

[37] A. R. Gilchrist, The Development and Breakaway of a Compressible Air Jet with Streamline Curvature and its Application to the Coanda, Durham University, Durham, UK, 1985.

[38] D. Ju-Long, "Control problems of grey systems," Systems \& Control Letters, vol. 1, no. 5, pp. 288-294, 1982.

[39] J. Zhou, J. Ren, and C. Yao, "Multi-objective optimization of multi-axis ball-end milling inconel 718 via grey relational analysis coupled with RBF neural network and PSO algorithm," Measurement, vol. 102, p. 15, 2017. 